\title{
Arithmetic Operations on Functions from Sets into Functional Sets
}

\author{
Artur Korniłowicz ${ }^{1}$ \\ Institute of Computer Science \\ University of Bialystok \\ Sosnowa 64, 15-887 Bialystok \\ Poland
}

\begin{abstract}
Summary. In this paper we introduce sets containing number-valued functions. Different arithmetic operations on maps between any set and such functional sets are later defined.
\end{abstract}

MML identifier: VALUED_2, version: $\underline{7.11 .01 \quad 4.117 .1046}$

The notation and terminology used here are introduced in the following papers: [4], [9], [10], [2], [11], [6], [3], [1], [8], [5], and [7].

\section{Functional SETS}

In this paper $x, X, X_{1}, X_{2}$ are sets.

Let $Y$ be a functional set. The functor $\operatorname{DOMS}(Y)$ is defined by:

(Def. 1) $\operatorname{DOMS}(Y)=\bigcup\{\operatorname{dom} f: f$ ranges over elements of $Y\}$.

Let us consider $X$. We say that $X$ is complex-functions-membered if and only if:

(Def. 2) If $x \in X$, then $x$ is a complex-valued function.

Let us consider $X$. We say that $X$ is extended-real-functions-membered if and only if:

(Def. 3) If $x \in X$, then $x$ is an extended real-valued function.

Let us consider $X$. We say that $X$ is real-functions-membered if and only if:

\footnotetext{
${ }^{1}$ The article was written while the author visited Shinshu University, Nagano, Japan.
} 
(Def. 4) If $x \in X$, then $x$ is a real-valued function.

Let us consider $X$. We say that $X$ is rational-functions-membered if and only if:

(Def. 5) If $x \in X$, then $x$ is a rational-valued function.

Let us consider $X$. We say that $X$ is integer-functions-membered if and only if:

(Def. 6) If $x \in X$, then $x$ is an integer-valued function.

Let us consider $X$. We say that $X$ is natural-functions-membered if and only if:

(Def. 7) If $x \in X$, then $x$ is a natural-valued function.

One can check the following observations:

* every set which is natural-functions-membered is also integer-functionsmembered,

* every set which is integer-functions-membered is also rational-functionsmembered,

* every set which is rational-functions-membered is also real-functionsmembered,

* every set which is real-functions-membered is also complex-functionsmembered, and

* every set which is real-functions-membered is also extended-realfunctions-membered.

Let us mention that every set which is empty is also natural-functionsmembered.

Let $f$ be a complex-valued function. Observe that $\{f\}$ is complex-functionsmembered.

One can verify that every set which is complex-functions-membered is also functional and every set which is extended-real-functions-membered is also functional.

One can verify that there exists a set which is natural-functions-membered and non empty.

Let $X$ be a complex-functions-membered set. One can verify that every subset of $X$ is complex-functions-membered.

Let $X$ be an extended-real-functions-membered set. Note that every subset of $X$ is extended-real-functions-membered.

Let $X$ be a real-functions-membered set. Note that every subset of $X$ is real-functions-membered.

Let $X$ be a rational-functions-membered set. Observe that every subset of $X$ is rational-functions-membered.

Let $X$ be an integer-functions-membered set. Note that every subset of $X$ is integer-functions-membered. 
Let $X$ be a natural-functions-membered set. Observe that every subset of $X$ is natural-functions-membered.

Let $D$ be a set. The functor $\mathbb{C}$-PFuncs $D$ yields a set and is defined by:

(Def. 8) For every set $f$ holds $f \in \mathbb{C}$-PFuncs $D$ iff $f$ is a partial function from $D$ to $\mathbb{C}$.

Let $D$ be a set. The functor $\mathbb{C}$-Funcs $D$ yielding a set is defined by:

(Def. 9) For every set $f$ holds $f \in \mathbb{C}$-Funcs $D$ iff $f$ is a function from $D$ into $\mathbb{C}$.

Let $D$ be a set. The functor $\overline{\mathbb{R}}$-PFuncs $D$ yields a set and is defined by:

(Def. 10) For every set $f$ holds $f \in \overline{\mathbb{R}}$-PFuncs $D$ iff $f$ is a partial function from $D$ to $\overline{\mathbb{R}}$.

Let $D$ be a set. The functor $\overline{\mathbb{R}}$-Funcs $D$ yields a set and is defined as follows:

(Def. 11) For every set $f$ holds $f \in \overline{\mathbb{R}}$-Funcs $D$ iff $f$ is a function from $D$ into $\overline{\mathbb{R}}$.

Let $D$ be a set. The functor $\mathbb{R}$-PFuncs $D$ yielding a set is defined by:

(Def. 12) For every set $f$ holds $f \in \mathbb{R}$-PFuncs $D$ iff $f$ is a partial function from $D$ to $\mathbb{R}$.

Let $D$ be a set. The functor $\mathbb{R}$-Funcs $D$ yielding a set is defined by:

(Def. 13) For every set $f$ holds $f \in \mathbb{R}$-Funcs $D$ iff $f$ is a function from $D$ into $\mathbb{R}$.

Let $D$ be a set. The functor $\mathbb{Q}$-PFuncs $D$ yields a set and is defined as follows:

(Def. 14) For every set $f$ holds $f \in \mathbb{Q}$-PFuncs $D$ iff $f$ is a partial function from $D$ to $\mathbb{Q}$.

Let $D$ be a set. The functor $\mathbb{Q}$-Funcs $D$ yields a set and is defined by:

(Def. 15) For every set $f$ holds $f \in \mathbb{Q}$-Funcs $D$ iff $f$ is a function from $D$ into $\mathbb{Q}$.

Let $D$ be a set. The functor $\mathbb{Z}$-PFuncs $D$ yielding a set is defined by:

(Def. 16) For every set $f$ holds $f \in \mathbb{Z}$-PFuncs $D$ iff $f$ is a partial function from $D$ to $\mathbb{Z}$.

Let $D$ be a set. The functor $\mathbb{Z}$-Funcs $D$ yields a set and is defined as follows:

(Def. 17) For every set $f$ holds $f \in \mathbb{Z}$-Funcs $D$ iff $f$ is a function from $D$ into $\mathbb{Z}$.

Let $D$ be a set. The functor $\mathbb{N}$-PFuncs $D$ yields a set and is defined by:

(Def. 18) For every set $f$ holds $f \in \mathbb{N}$-PFuncs $D$ iff $f$ is a partial function from $D$ to $\mathbb{N}$.

Let $D$ be a set. The functor $\mathbb{N}$-Funcs $D$ yielding a set is defined by:

(Def. 19) For every set $f$ holds $f \in \mathbb{N}$-Funcs $D$ iff $f$ is a function from $D$ into $\mathbb{N}$.

The following propositions are true:

(1) $\mathbb{C}$-Funcs $X$ is a subset of $\mathbb{C}$-PFuncs $X$.

(2) $\overline{\mathbb{R}}$-Funcs $X$ is a subset of $\overline{\mathbb{R}}$-PFuncs $X$.

(3) $\mathbb{R}$-Funcs $X$ is a subset of $\mathbb{R}$-PFuncs $X$.

(4) $\mathbb{Q}$-Funcs $X$ is a subset of $\mathbb{Q}$-PFuncs $X$.

(5) $\mathbb{Z}$-Funcs $X$ is a subset of $\mathbb{Z}$-PFuncs $X$. 
(6) $\mathbb{N}$-Funcs $X$ is a subset of $\mathbb{N}$-PFuncs $X$.

Let us consider $X$. One can verify the following observations:

* $\mathbb{C}$-PFuncs $X$ is complex-functions-membered,

* $\mathbb{C}$-Funcs $X$ is complex-functions-membered,

* $\overline{\mathbb{R}}$-PFuncs $X$ is extended-real-functions-membered,

* $\overline{\mathbb{R}}$-Funcs $X$ is extended-real-functions-membered,

* $\mathbb{R}$-PFuncs $X$ is real-functions-membered,

* $\mathbb{R}$-Funcs $X$ is real-functions-membered,

* $\mathbb{Q}$-PFuncs $X$ is rational-functions-membered,

* $\mathbb{Q}$-Funcs $X$ is rational-functions-membered,

* $\mathbb{Z}$-PFuncs $X$ is integer-functions-membered,

* $\mathbb{Z}$-Funcs $X$ is integer-functions-membered,

* $\quad \mathbb{N}$-PFuncs $X$ is natural-functions-membered, and

* $\mathbb{N}$-Funcs $X$ is natural-functions-membered.

Let $X$ be a complex-functions-membered set. Observe that every element of $X$ is complex-valued.

Let $X$ be an extended-real-functions-membered set. One can check that every element of $X$ is extended real-valued.

Let $X$ be a real-functions-membered set. One can check that every element of $X$ is real-valued.

Let $X$ be a rational-functions-membered set. One can check that every element of $X$ is rational-valued.

Let $X$ be an integer-functions-membered set. Observe that every element of $X$ is integer-valued.

Let $X$ be a natural-functions-membered set. Observe that every element of $X$ is natural-valued.

Let $X, x$ be sets, let $Y$ be a complex-functions-membered set, and let $f$ be a partial function from $X$ to $Y$. Observe that $f(x)$ is function-like and relationlike.

Let $X, x$ be sets, let $Y$ be an extended-real-functions-membered set, and let $f$ be a partial function from $X$ to $Y$. Observe that $f(x)$ is function-like and relation-like.

Let us consider $X, x$, let $Y$ be a complex-functions-membered set, and let $f$ be a partial function from $X$ to $Y$. One can check that $f(x)$ is complex-valued.

Let us consider $X, x$, let $Y$ be an extended-real-functions-membered set, and let $f$ be a partial function from $X$ to $Y$. One can verify that $f(x)$ is extended real-valued.

Let us consider $X, x$, let $Y$ be a real-functions-membered set, and let $f$ be a partial function from $X$ to $Y$. Note that $f(x)$ is real-valued. 
Let us consider $X, x$, let $Y$ be a rational-functions-membered set, and let $f$ be a partial function from $X$ to $Y$. Note that $f(x)$ is rational-valued.

Let us consider $X, x$, let $Y$ be an integer-functions-membered set, and let $f$ be a partial function from $X$ to $Y$. Note that $f(x)$ is integer-valued.

Let us consider $X, x$, let $Y$ be a natural-functions-membered set, and let $f$ be a partial function from $X$ to $Y$. One can check that $f(x)$ is natural-valued.

Let us consider $X$ and let $Y$ be a complex-membered set. One can check that $X \dot{\rightarrow} Y$ is complex-functions-membered.

Let us consider $X$ and let $Y$ be an extended real-membered set. Observe that $X \dot{\rightarrow} Y$ is extended-real-functions-membered.

Let us consider $X$ and let $Y$ be a real-membered set. Observe that $X \dot{\rightarrow} Y$ is real-functions-membered.

Let us consider $X$ and let $Y$ be a rational-membered set. Observe that $X \dot{\rightarrow} Y$ is rational-functions-membered.

Let us consider $X$ and let $Y$ be an integer-membered set. Observe that $X \dot{\rightarrow} Y$ is integer-functions-membered.

Let us consider $X$ and let $Y$ be a natural-membered set. One can verify that $X \dot{\rightarrow} Y$ is natural-functions-membered.

Let us consider $X$ and let $Y$ be a complex-membered set. Note that $Y^{X}$ is complex-functions-membered.

Let us consider $X$ and let $Y$ be an extended real-membered set. Note that $Y^{X}$ is extended-real-functions-membered.

Let us consider $X$ and let $Y$ be a real-membered set. Note that $Y^{X}$ is realfunctions-membered.

Let us consider $X$ and let $Y$ be a rational-membered set. Note that $Y^{X}$ is rational-functions-membered.

Let us consider $X$ and let $Y$ be an integer-membered set. Note that $Y^{X}$ is integer-functions-membered.

Let us consider $X$ and let $Y$ be a natural-membered set. One can check that $Y^{X}$ is natural-functions-membered.

Let $R$ be a binary relation. We say that $R$ is complex-functions-valued if and only if:

(Def. 20) $\operatorname{rng} R$ is complex-functions-membered.

We say that $R$ is extended-real-functions-valued if and only if:

(Def. 21) $\operatorname{rng} R$ is extended-real-functions-membered.

We say that $R$ is real-functions-valued if and only if:

(Def. 22) $\operatorname{rng} R$ is real-functions-membered.

We say that $R$ is rational-functions-valued if and only if:

(Def. 23) $\operatorname{rng} R$ is rational-functions-membered.

We say that $R$ is integer-functions-valued if and only if: 
(Def. 24) $\operatorname{rng} R$ is integer-functions-membered.

We say that $R$ is natural-functions-valued if and only if:

(Def. 25) $\operatorname{rng} R$ is natural-functions-membered.

Let $f$ be a function. Let us observe that $f$ is complex-functions-valued if and only if:

(Def. 26) For every set $x$ such that $x \in \operatorname{dom} f$ holds $f(x)$ is a complex-valued function.

Let us observe that $f$ is extended-real-functions-valued if and only if:

(Def. 27) For every set $x$ such that $x \in \operatorname{dom} f$ holds $f(x)$ is an extended real-valued function.

Let us observe that $f$ is real-functions-valued if and only if:

(Def. 28) For every set $x$ such that $x \in \operatorname{dom} f$ holds $f(x)$ is a real-valued function.

Let us observe that $f$ is rational-functions-valued if and only if:

(Def. 29) For every set $x$ such that $x \in \operatorname{dom} f$ holds $f(x)$ is a rational-valued function.

Let us observe that $f$ is integer-functions-valued if and only if:

(Def. 30) For every set $x$ such that $x \in \operatorname{dom} f$ holds $f(x)$ is an integer-valued function.

Let us observe that $f$ is natural-functions-valued if and only if:

(Def. 31) For every set $x$ such that $x \in \operatorname{dom} f$ holds $f(x)$ is a natural-valued function.

One can verify the following observations:

* every binary relation which is natural-functions-valued is also integerfunctions-valued,

* every binary relation which is integer-functions-valued is also rationalfunctions-valued,

* every binary relation which is rational-functions-valued is also realfunctions-valued,

* every binary relation which is real-functions-valued is also extended-realfunctions-valued, and

* every binary relation which is real-functions-valued is also complexfunctions-valued.

Let us note that every binary relation which is empty is also naturalfunctions-valued.

Let us mention that there exists a function which is natural-functions-valued.

Let $R$ be a complex-functions-valued binary relation. Note that $\operatorname{rng} R$ is complex-functions-membered.

Let $R$ be an extended-real-functions-valued binary relation. Observe that $\operatorname{rng} R$ is extended-real-functions-membered. 
Let $R$ be a real-functions-valued binary relation. Note that $\operatorname{rng} R$ is realfunctions-membered.

Let $R$ be a rational-functions-valued binary relation. Observe that $\operatorname{rng} R$ is rational-functions-membered.

Let $R$ be an integer-functions-valued binary relation. One can verify that $\operatorname{rng} R$ is integer-functions-membered.

Let $R$ be a natural-functions-valued binary relation. One can check that $\operatorname{rng} R$ is natural-functions-membered.

Let us consider $X$ and let $Y$ be a complex-functions-membered set. Observe that every partial function from $X$ to $Y$ is complex-functions-valued.

Let us consider $X$ and let $Y$ be an extended-real-functions-membered set. One can check that every partial function from $X$ to $Y$ is extended-realfunctions-valued.

Let us consider $X$ and let $Y$ be a real-functions-membered set. One can check that every partial function from $X$ to $Y$ is real-functions-valued.

Let us consider $X$ and let $Y$ be a rational-functions-membered set. Observe that every partial function from $X$ to $Y$ is rational-functions-valued.

Let us consider $X$ and let $Y$ be an integer-functions-membered set. Observe that every partial function from $X$ to $Y$ is integer-functions-valued.

Let us consider $X$ and let $Y$ be a natural-functions-membered set. Note that every partial function from $X$ to $Y$ is natural-functions-valued.

Let $f$ be a complex-functions-valued function and let us consider $x$. Note that $f(x)$ is function-like and relation-like.

Let $f$ be an extended-real-functions-valued function and let us consider $x$. Observe that $f(x)$ is function-like and relation-like.

Let $f$ be a complex-functions-valued function and let us consider $x$. One can verify that $f(x)$ is complex-valued.

Let $f$ be an extended-real-functions-valued function and let us consider $x$. Note that $f(x)$ is extended real-valued.

Let $f$ be a real-functions-valued function and let us consider $x$. One can verify that $f(x)$ is real-valued.

Let $f$ be a rational-functions-valued function and let us consider $x$. Observe that $f(x)$ is rational-valued.

Let $f$ be an integer-functions-valued function and let us consider $x$. Note that $f(x)$ is integer-valued.

Let $f$ be a natural-functions-valued function and let us consider $x$. One can check that $f(x)$ is natural-valued.

\section{Operations}

For simplicity, we adopt the following rules: $Y, Y_{1}, Y_{2}$ are complex-functionsmembered sets, $c, c_{1}, c_{2}$ are complex numbers, $f$ is a partial function from $X$ 
to $Y, f_{1}$ is a partial function from $X_{1}$ to $Y_{1}, f_{2}$ is a partial function from $X_{2}$ to $Y_{2}$, and $g, h, k$ are complex-valued functions.

We now state a number of propositions:

(7) If $g \neq \emptyset$ and $g+c_{1}=g+c_{2}$, then $c_{1}=c_{2}$.

(8) If $g \neq \emptyset$ and $g-c_{1}=g-c_{2}$, then $c_{1}=c_{2}$.

(9) If $g \neq \emptyset$ and $g$ is non-empty and $g c_{1}=g c_{2}$, then $c_{1}=c_{2}$.

(10) $-(g+c)=-g-c$.

(11) $-(g-c)=-g+c$.

(12) $\left(g+c_{1}\right)+c_{2}=g+\left(c_{1}+c_{2}\right)$.

(13) $\left(g+c_{1}\right)-c_{2}=g+\left(c_{1}-c_{2}\right)$.

(14) $\left(g-c_{1}\right)+c_{2}=g-\left(c_{1}-c_{2}\right)$.

(15) $g-c_{1}-c_{2}=g-\left(c_{1}+c_{2}\right)$.

(16) $g c_{1} c_{2}=g\left(c_{1} \cdot c_{2}\right)$.

(17) $-(g+h)=-g-h$.

(18) $g-h=-(h-g)$.

(19) $(g h) / k=g(h / k)$.

(20) $(g / h) k=(g k) / h$.

(21) $g / h / k=g /(h k)$.

(22) $c-g=(-c) g$.

(23) $c-g=-c g$.

(24) $(-c) g=-c g$.

(25) $-g h=(-g) h$.

(26) $-g / h=(-g) / h$.

(27) $-g / h=g /-h$.

Let $f$ be a complex-valued function and let $c$ be a complex number. The functor $f / c$ yields a function and is defined as follows:

(Def. 32) $f / c=\frac{1}{c} f$.

Let $f$ be a complex-valued function and let $c$ be a complex number. Note that $f / c$ is complex-valued.

Let $f$ be a real-valued function and let $r$ be a real number. Note that $f / r$ is real-valued.

Let $f$ be a rational-valued function and let $r$ be a rational number. One can check that $f / r$ is rational-valued.

Let $f$ be a complex-valued finite sequence and let $c$ be a complex number. One can check that $f / c$ is finite sequence-like.

The following propositions are true:

(28) $\operatorname{dom}(g / c)=\operatorname{dom} g$.

(29) $(g / c)(x)=\frac{g(x)}{c}$. 
(30) $(-g) / c=-g / c$.

(31) $g /-c=-g / c$.

(32) $g /-c=(-g) / c$.

(33) If $g \neq \emptyset$ and $g$ is non-empty and $g / c_{1}=g / c_{2}$, then $c_{1}=c_{2}$.

(34) $\left(g c_{1}\right) / c_{2}=g \frac{c_{1}}{c_{2}}$.

(35) $\left(g / c_{1}\right) c_{2}=\left(g c_{2}\right) / c_{1}$.

(36) $g / c_{1} / c_{2}=g /\left(c_{1} \cdot c_{2}\right)$.

(37) $(g+h) / c=g / c+h / c$.

(38) $(g-h) / c=g / c-h / c$.

(39) $(g h) / c=g(h / c)$.

(40) $(g / h) / c=g /(h c)$.

Let us consider $X$, let $Y$ be a complex-functions-membered set, and let $f$ be a partial function from $X$ to $Y$. The functor $-f$ yields a function and is defined by:

(Def. 33) $\operatorname{dom}(-f)=\operatorname{dom} f$ and for every set $x$ such that $x \in \operatorname{dom}(-f)$ holds $(-f)(x)=-f(x)$.

Let us consider $X$, let $Y$ be a complex-functions-membered set, and let $f$ be a partial function from $X$ to $Y$. Then $-f$ is a partial function from $X$ to $\mathbb{C}$-PFuncs DOMS $(Y)$.

Let us consider $X$, let $Y$ be a real-functions-membered set, and let $f$ be a partial function from $X$ to $Y$. Then $-f$ is a partial function from $X$ to $\mathbb{R}$-PFuncs DOMS $(Y)$.

Let us consider $X$, let $Y$ be a rational-functions-membered set, and let $f$ be a partial function from $X$ to $Y$. Then $-f$ is a partial function from $X$ to $\mathbb{Q}$-PFuncs DOMS $(Y)$.

Let us consider $X$, let $Y$ be an integer-functions-membered set, and let $f$ be a partial function from $X$ to $Y$. Then $-f$ is a partial function from $X$ to $\mathbb{Z}$-PFuncs $\operatorname{DOMS}(Y)$.

Let $Y$ be a complex-functions-membered set and let $f$ be a finite sequence of elements of $Y$. One can check that $-f$ is finite sequence-like.

We now state two propositions:

(41) $--f=f$.

(42) If $-f_{1}=-f_{2}$, then $f_{1}=f_{2}$.

Let $X$ be a complex-functions-membered set, let $Y$ be a set, and let $f$ be a partial function from $X$ to $Y$. The functor $f \circ-$ yielding a function is defined as follows:

(Def. 34) $\operatorname{dom}(f \circ-)=\operatorname{dom} f$ and for every complex-valued function $x$ such that $x \in \operatorname{dom}(f \circ-)$ holds $(f \circ-)(x)=f(-x)$. 
Let us consider $X$, let $Y$ be a complex-functions-membered set, and let $f$ be a partial function from $X$ to $Y$. The functor ${ }^{1} / f$ yields a function and is defined as follows:

(Def. 35) $\operatorname{dom}^{1} / f=\operatorname{dom} f$ and for every set $x$ such that $x \in \operatorname{dom}^{1} / f$ holds $(1 / f)(x)=f(x)^{-1}$.

Let us consider $X$, let $Y$ be a complex-functions-membered set, and let $f$ be a partial function from $X$ to $Y$. Then ${ }^{1} / f$ is a partial function from $X$ to C-PFuncs $\operatorname{DOMS}(Y)$.

Let us consider $X$, let $Y$ be a real-functions-membered set, and let $f$ be a partial function from $X$ to $Y$. Then ${ }^{1} / f$ is a partial function from $X$ to $\mathbb{R}$-PFuncs DOMS $(Y)$.

Let us consider $X$, let $Y$ be a rational-functions-membered set, and let $f$ be a partial function from $X$ to $Y$. Then ${ }^{1} / f$ is a partial function from $X$ to $\mathbb{Q}$-PFuncs DOMS $(Y)$.

Let $Y$ be a complex-functions-membered set and let $f$ be a finite sequence of elements of $Y$. Note that ${ }^{1} / f$ is finite sequence-like.

The following proposition is true

(43) $1 / 1 / f=f$.

Let us consider $X$, let $Y$ be a complex-functions-membered set, and let $f$ be a partial function from $X$ to $Y$. The functor $|f|$ yields a function and is defined by:

(Def. 36) $\operatorname{dom}|f|=\operatorname{dom} f$ and for every set $x$ such that $x \in \operatorname{dom}|f|$ holds $|f|(x)=$ $|f(x)|$.

Let us consider $X$, let $Y$ be a complex-functions-membered set, and let $f$ be a partial function from $X$ to $Y$. Then $|f|$ is a partial function from $X$ to $\mathbb{C}$-PFuncs $\operatorname{DOMS}(Y)$.

Let us consider $X$, let $Y$ be a real-functions-membered set, and let $f$ be a partial function from $X$ to $Y$. Then $|f|$ is a partial function from $X$ to $\mathbb{R}$-PFuncs DOMS $(Y)$.

Let us consider $X$, let $Y$ be a rational-functions-membered set, and let $f$ be a partial function from $X$ to $Y$. Then $|f|$ is a partial function from $X$ to $\mathbb{Q}$-PFuncs DOMS $(Y)$.

Let us consider $X$, let $Y$ be an integer-functions-membered set, and let $f$ be a partial function from $X$ to $Y$. Then $|f|$ is a partial function from $X$ to $\mathbb{N}$-PFuncs DOMS $(Y)$.

Let $Y$ be a complex-functions-membered set and let $f$ be a finite sequence of elements of $Y$. Note that $|f|$ is finite sequence-like.

We now state the proposition

(44) $\quad\|f\|=|f|$.

Let us consider $X$, let $Y$ be a complex-functions-membered set, let $f$ be a partial function from $X$ to $Y$, and let $c$ be a complex number. The functor $f+c$ 
yields a function and is defined by:

(Def. 37) $\operatorname{dom}(f+c)=\operatorname{dom} f$ and for every set $x$ such that $x \in \operatorname{dom}(f+c)$ holds $(f+c)(x)=c+f(x)$.

Let us consider $X$, let $Y$ be a complex-functions-membered set, let $f$ be a partial function from $X$ to $Y$, and let $c$ be a complex number. Then $f+c$ is a partial function from $X$ to $\mathbb{C}$-PFuncs $\operatorname{DOMS}(Y)$.

Let us consider $X$, let $Y$ be a real-functions-membered set, let $f$ be a partial function from $X$ to $Y$, and let $c$ be a real number. Then $f+c$ is a partial function from $X$ to $\mathbb{R}$-PFuncs $\operatorname{DOMS}(Y)$.

Let us consider $X$, let $Y$ be a rational-functions-membered set, let $f$ be a partial function from $X$ to $Y$, and let $c$ be a rational number. Then $f+c$ is a partial function from $X$ to $\mathbb{Q}$-PFuncs $\operatorname{DOMS}(Y)$.

Let us consider $X$, let $Y$ be an integer-functions-membered set, let $f$ be a partial function from $X$ to $Y$, and let $c$ be an integer number. Then $f+c$ is a partial function from $X$ to $\mathbb{Z}$-PFuncs $\operatorname{DOMS}(Y)$.

Let us consider $X$, let $Y$ be a natural-functions-membered set, let $f$ be a partial function from $X$ to $Y$, and let $c$ be a natural number. Then $f+c$ is a partial function from $X$ to $\mathbb{N}$-PFuncs $\operatorname{DOMS}(Y)$.

One can prove the following propositions:

(45) $f+c_{1}+c_{2}=f+\left(c_{1}+c_{2}\right)$.

(46) If $f \neq \emptyset$ and $f$ is non-empty and $f+c_{1}=f+c_{2}$, then $c_{1}=c_{2}$.

Let us consider $X$, let $Y$ be a complex-functions-membered set, let $f$ be a partial function from $X$ to $Y$, and let $c$ be a complex number. The functor $f-c$ yields a function and is defined as follows:

(Def. 38) $f-c=f+-c$.

We now state two propositions:

(47) $\operatorname{dom}(f-c)=\operatorname{dom} f$.

(48) If $x \in \operatorname{dom}(f-c)$, then $(f-c)(x)=f(x)-c$.

Let us consider $X$, let $Y$ be a complex-functions-membered set, let $f$ be a partial function from $X$ to $Y$, and let $c$ be a complex number. Then $f-c$ is a partial function from $X$ to $\mathbb{C}$-PFuncs $\operatorname{DOMS}(Y)$.

Let us consider $X$, let $Y$ be a real-functions-membered set, let $f$ be a partial function from $X$ to $Y$, and let $c$ be a real number. Then $f-c$ is a partial function from $X$ to $\mathbb{R}$-PFuncs $\operatorname{DOMS}(Y)$.

Let us consider $X$, let $Y$ be a rational-functions-membered set, let $f$ be a partial function from $X$ to $Y$, and let $c$ be a rational number. Then $f-c$ is a partial function from $X$ to $\mathbb{Q}$-PFuncs $\operatorname{DOMS}(Y)$.

Let us consider $X$, let $Y$ be an integer-functions-membered set, let $f$ be a partial function from $X$ to $Y$, and let $c$ be an integer number. Then $f-c$ is a partial function from $X$ to $\mathbb{Z}$-PFuncs $\operatorname{DOMS}(Y)$. 
We now state four propositions:

(49) If $f \neq \emptyset$ and $f$ is non-empty and $f-c_{1}=f-c_{2}$, then $c_{1}=c_{2}$.

(50) $\left(f+c_{1}\right)-c_{2}=f+\left(c_{1}-c_{2}\right)$.

(51) $\left(f-c_{1}\right)+c_{2}=f-\left(c_{1}-c_{2}\right)$.

(52) $f-c_{1}-c_{2}=f-\left(c_{1}+c_{2}\right)$.

Let us consider $X$, let $Y$ be a complex-functions-membered set, let $f$ be a partial function from $X$ to $Y$, and let $c$ be a complex number. The functor $f \cdot c$ yielding a function is defined as follows:

(Def. 39) $\operatorname{dom}(f \cdot c)=\operatorname{dom} f$ and for every set $x$ such that $x \in \operatorname{dom}(f \cdot c)$ holds $(f \cdot c)(x)=c f(x)$.

Let us consider $X$, let $Y$ be a complex-functions-membered set, let $f$ be a partial function from $X$ to $Y$, and let $c$ be a complex number. Then $f \cdot c$ is a partial function from $X$ to $\mathbb{C}$-PFuncs $\operatorname{DOMS}(Y)$.

Let us consider $X$, let $Y$ be a real-functions-membered set, let $f$ be a partial function from $X$ to $Y$, and let $c$ be a real number. Then $f \cdot c$ is a partial function from $X$ to $\mathbb{R}$-PFuncs $\operatorname{DOMS}(Y)$.

Let us consider $X$, let $Y$ be a rational-functions-membered set, let $f$ be a partial function from $X$ to $Y$, and let $c$ be a rational number. Then $f \cdot c$ is a partial function from $X$ to $\mathbb{Q}$-PFuncs $\operatorname{DOMS}(Y)$.

Let us consider $X$, let $Y$ be an integer-functions-membered set, let $f$ be a partial function from $X$ to $Y$, and let $c$ be an integer number. Then $f \cdot c$ is a partial function from $X$ to $\mathbb{Z}$-PFuncs $\operatorname{DOMS}(Y)$.

Let us consider $X$, let $Y$ be a natural-functions-membered set, let $f$ be a partial function from $X$ to $Y$, and let $c$ be a natural number. Then $f \cdot c$ is a partial function from $X$ to $\mathbb{N}$-PFuncs $\operatorname{DOMS}(Y)$.

The following two propositions are true:

(53) $f \cdot c_{1} \cdot c_{2}=f \cdot\left(c_{1} \cdot c_{2}\right)$.

(54) If $f \neq \emptyset$ and $f$ is non-empty and for every $x$ such that $x \in \operatorname{dom} f$ holds $f(x)$ is non-empty and $f \cdot c_{1}=f \cdot c_{2}$, then $c_{1}=c_{2}$.

Let us consider $X$, let $Y$ be a complex-functions-membered set, let $f$ be a partial function from $X$ to $Y$, and let $c$ be a complex number. The functor $f / c$ yielding a function is defined as follows:

(Def. 40) $f / c=f \cdot c^{-1}$.

One can prove the following propositions:

(55) $\operatorname{dom}(f / c)=\operatorname{dom} f$.

(56) If $x \in \operatorname{dom}(f / c)$, then $(f / c)(x)=c^{-1} f(x)$.

Let us consider $X$, let $Y$ be a complex-functions-membered set, let $f$ be a partial function from $X$ to $Y$, and let $c$ be a complex number. Then $f / c$ is a partial function from $X$ to $\mathbb{C}$-PFuncs $\operatorname{DOMS}(Y)$. 
Let us consider $X$, let $Y$ be a real-functions-membered set, let $f$ be a partial function from $X$ to $Y$, and let $c$ be a real number. Then $f / c$ is a partial function from $X$ to $\mathbb{R}$-PFuncs $\operatorname{DOMS}(Y)$.

Let us consider $X$, let $Y$ be a rational-functions-membered set, let $f$ be a partial function from $X$ to $Y$, and let $c$ be a rational number. Then $f / c$ is a partial function from $X$ to $\mathbb{Q}$-PFuncs $\operatorname{DOMS}(Y)$.

The following propositions are true:

(57) $f / c_{1} / c_{2}=f /\left(c_{1} \cdot c_{2}\right)$.

(58) If $f \neq \emptyset$ and $f$ is non-empty and for every $x$ such that $x \in \operatorname{dom} f$ holds $f(x)$ is non-empty and $f / c_{1}=f / c_{2}$, then $c_{1}=c_{2}$.

Let us consider $X$, let $Y$ be a complex-functions-membered set, let $f$ be a partial function from $X$ to $Y$, and let $g$ be a complex-valued function. The functor $f+g$ yielding a function is defined as follows:

(Def. 41) $\operatorname{dom}(f+g)=\operatorname{dom} f \cap \operatorname{dom} g$ and for every set $x$ such that $x \in \operatorname{dom}(f+g)$ holds $(f+g)(x)=f(x)+g(x)$.

Let us consider $X$, let $Y$ be a complex-functions-membered set, let $f$ be a partial function from $X$ to $Y$, and let $g$ be a complex-valued function. Then $f+g$ is a partial function from $X \cap \operatorname{dom} g$ to $\mathbb{C}$-PFuncs $\operatorname{DOMS}(Y)$.

Let us consider $X$, let $Y$ be a real-functions-membered set, let $f$ be a partial function from $X$ to $Y$, and let $g$ be a real-valued function. Then $f+g$ is a partial function from $X \cap \operatorname{dom} g$ to $\mathbb{R}$-PFuncs $\operatorname{DOMS}(Y)$.

Let us consider $X$, let $Y$ be a rational-functions-membered set, let $f$ be a partial function from $X$ to $Y$, and let $g$ be a rational-valued function. Then $f+g$ is a partial function from $X \cap \operatorname{dom} g$ to $\mathbb{Q}$-PFuncs $\operatorname{DOMS}(Y)$.

Let us consider $X$, let $Y$ be an integer-functions-membered set, let $f$ be a partial function from $X$ to $Y$, and let $g$ be an integer-valued function. Then $f+g$ is a partial function from $X \cap \operatorname{dom} g$ to $\mathbb{Z}$-PFuncs $\operatorname{DOMS}(Y)$.

Let us consider $X$, let $Y$ be a natural-functions-membered set, let $f$ be a partial function from $X$ to $Y$, and let $g$ be a natural-valued function. Then $f+g$ is a partial function from $X \cap \operatorname{dom} g$ to $\mathbb{N}$-PFuncs $\operatorname{DOMS}(Y)$.

Next we state two propositions:

(59) $f+g+h=f+(g+h)$.

(60) $-(f+g)=(-f)+-g$.

Let us consider $X$, let $Y$ be a complex-functions-membered set, let $f$ be a partial function from $X$ to $Y$, and let $g$ be a complex-valued function. The functor $f-g$ yields a function and is defined by:

(Def. 42) $f-g=f+-g$.

We now state two propositions:

(61) $\operatorname{dom}(f-g)=\operatorname{dom} f \cap \operatorname{dom} g$.

(62) If $x \in \operatorname{dom}(f-g)$, then $(f-g)(x)=f(x)-g(x)$. 
Let us consider $X$, let $Y$ be a complex-functions-membered set, let $f$ be a partial function from $X$ to $Y$, and let $g$ be a complex-valued function. Then $f-g$ is a partial function from $X \cap \operatorname{dom} g$ to $\mathbb{C}$-PFuncs $\operatorname{DOMS}(Y)$.

Let us consider $X$, let $Y$ be a real-functions-membered set, let $f$ be a partial function from $X$ to $Y$, and let $g$ be a real-valued function. Then $f-g$ is a partial function from $X \cap \operatorname{dom} g$ to $\mathbb{R}$-PFuncs $\operatorname{DOMS}(Y)$.

Let us consider $X$, let $Y$ be a rational-functions-membered set, let $f$ be a partial function from $X$ to $Y$, and let $g$ be a rational-valued function. Then $f-g$ is a partial function from $X \cap \operatorname{dom} g$ to $\mathbb{Q}$-PFuncs $\operatorname{DOMS}(Y)$.

Let us consider $X$, let $Y$ be an integer-functions-membered set, let $f$ be a partial function from $X$ to $Y$, and let $g$ be an integer-valued function. Then $f-g$ is a partial function from $X \cap \operatorname{dom} g$ to $\mathbb{Z}$-PFuncs $\operatorname{DOMS}(Y)$.

The following propositions are true:

(63) $f--g=f+g$.

(64) $-(f-g)=(-f)+g$.

(65) $(f+g)-h=f+(g-h)$.

(66) $(f-g)+h=f-(g-h)$.

(67) $f-g-h=f-(g+h)$.

Let us consider $X$, let $Y$ be a complex-functions-membered set, let $f$ be a partial function from $X$ to $Y$, and let $g$ be a complex-valued function. The functor $f \cdot g$ yielding a function is defined by:

(Def. 43) $\operatorname{dom}(f \cdot g)=\operatorname{dom} f \cap \operatorname{dom} g$ and for every set $x$ such that $x \in \operatorname{dom}(f \cdot g)$ holds $(f \cdot g)(x)=f(x) g(x)$.

Let us consider $X$, let $Y$ be a complex-functions-membered set, let $f$ be a partial function from $X$ to $Y$, and let $g$ be a complex-valued function. Then $f \cdot g$ is a partial function from $X \cap \operatorname{dom} g$ to $\mathbb{C}$-PFuncs $\operatorname{DOMS}(Y)$.

Let us consider $X$, let $Y$ be a real-functions-membered set, let $f$ be a partial function from $X$ to $Y$, and let $g$ be a real-valued function. Then $f \cdot g$ is a partial function from $X \cap \operatorname{dom} g$ to $\mathbb{R}$-PFuncs $\operatorname{DOMS}(Y)$.

Let us consider $X$, let $Y$ be a rational-functions-membered set, let $f$ be a partial function from $X$ to $Y$, and let $g$ be a rational-valued function. Then $f \cdot g$ is a partial function from $X \cap \operatorname{dom} g$ to $\mathbb{Q}$-PFuncs $\operatorname{DOMS}(Y)$.

Let us consider $X$, let $Y$ be an integer-functions-membered set, let $f$ be a partial function from $X$ to $Y$, and let $g$ be an integer-valued function. Then $f \cdot g$ is a partial function from $X \cap \operatorname{dom} g$ to $\mathbb{Z}$-PFuncs $\operatorname{DOMS}(Y)$.

Let us consider $X$, let $Y$ be a natural-functions-membered set, let $f$ be a partial function from $X$ to $Y$, and let $g$ be a natural-valued function. Then $f \cdot g$ is a partial function from $X \cap \operatorname{dom} g$ to $\mathbb{N}$-PFuncs $\operatorname{DOMS}(Y)$.

Next we state three propositions:

(68) $f \cdot-g=(-f) \cdot g$. 
(69) $f \cdot-g=-f \cdot g$.

(70) $f \cdot g \cdot h=f \cdot(g h)$.

Let us consider $X$, let $Y$ be a complex-functions-membered set, let $f$ be a partial function from $X$ to $Y$, and let $g$ be a complex-valued function. The functor $f / g$ yields a function and is defined by:

(Def. 44) $f / g=f \cdot g^{-1}$.

Next we state two propositions:

(71) $\operatorname{dom}(f / g)=\operatorname{dom} f \cap \operatorname{dom} g$.

(72) If $x \in \operatorname{dom}(f / g)$, then $(f / g)(x)=f(x) / g(x)$.

Let us consider $X$, let $Y$ be a complex-functions-membered set, let $f$ be a partial function from $X$ to $Y$, and let $g$ be a complex-valued function. Then $f / g$ is a partial function from $X \cap \operatorname{dom} g$ to $\mathbb{C}$-PFuncs $\operatorname{DOMS}(Y)$.

Let us consider $X$, let $Y$ be a real-functions-membered set, let $f$ be a partial function from $X$ to $Y$, and let $g$ be a real-valued function. Then $f / g$ is a partial function from $X \cap \operatorname{dom} g$ to $\mathbb{R}$-PFuncs $\operatorname{DOMS}(Y)$.

Let us consider $X$, let $Y$ be a rational-functions-membered set, let $f$ be a partial function from $X$ to $Y$, and let $g$ be a rational-valued function. Then $f / g$ is a partial function from $X \cap \operatorname{dom} g$ to $\mathbb{Q}$-PFuncs $\operatorname{DOMS}(Y)$.

Next we state the proposition

(73) $(f \cdot g) / h=f \cdot(g / h)$.

Let $X_{1}, X_{2}$ be sets, let $Y_{1}, Y_{2}$ be complex-functions-membered sets, let $f$ be a partial function from $X_{1}$ to $Y_{1}$, and let $g$ be a partial function from $X_{2}$ to $Y_{2}$. The functor $f+g$ yielding a function is defined as follows:

(Def. 45) $\operatorname{dom}(f+g)=\operatorname{dom} f \cap \operatorname{dom} g$ and for every set $x$ such that $x \in \operatorname{dom}(f+g)$ holds $(f+g)(x)=f(x)+g(x)$.

Let $X_{1}, X_{2}$ be sets, let $Y_{1}, Y_{2}$ be complex-functions-membered sets, let $f$ be a partial function from $X_{1}$ to $Y_{1}$, and let $g$ be a partial function from $X_{2}$ to $Y_{2}$. Then $f+g$ is a partial function from $X_{1} \cap X_{2}$ to $\mathbb{C}$-PFuncs(DOMS $\left(Y_{1}\right) \cap$ $\left.\operatorname{DOMS}\left(Y_{2}\right)\right)$.

Let $X_{1}, X_{2}$ be sets, let $Y_{1}, Y_{2}$ be real-functions-membered sets, let $f$ be a partial function from $X_{1}$ to $Y_{1}$, and let $g$ be a partial function from $X_{2}$ to $Y_{2}$. Then $f+g$ is a partial function from $X_{1} \cap X_{2}$ to $\mathbb{R}-\operatorname{PFuncs}\left(\operatorname{DOMS}\left(Y_{1}\right) \cap\right.$ $\left.\operatorname{DOMS}\left(Y_{2}\right)\right)$.

Let $X_{1}, X_{2}$ be sets, let $Y_{1}, Y_{2}$ be rational-functions-membered sets, let $f$ be a partial function from $X_{1}$ to $Y_{1}$, and let $g$ be a partial function from $X_{2}$ to $Y_{2}$. Then $f+g$ is a partial function from $X_{1} \cap X_{2}$ to $\mathbb{Q}$-PFuncs(DOMS $\left(Y_{1}\right) \cap$ $\left.\operatorname{DOMS}\left(Y_{2}\right)\right)$.

Let $X_{1}, X_{2}$ be sets, let $Y_{1}, Y_{2}$ be integer-functions-membered sets, let $f$ be a partial function from $X_{1}$ to $Y_{1}$, and let $g$ be a partial function from $X_{2}$ to 
$Y_{2}$. Then $f+g$ is a partial function from $X_{1} \cap X_{2}$ to $\mathbb{Z}$-PFuncs(DOMS $\left(Y_{1}\right) \cap$ $\left.\operatorname{DOMS}\left(Y_{2}\right)\right)$.

Let $X_{1}, X_{2}$ be sets, let $Y_{1}, Y_{2}$ be natural-functions-membered sets, let $f$ be a partial function from $X_{1}$ to $Y_{1}$, and let $g$ be a partial function from $X_{2}$ to $Y_{2}$. Then $f+g$ is a partial function from $X_{1} \cap X_{2}$ to $\mathbb{N}$-PFuncs(DOMS $\left(Y_{1}\right) \cap$ $\left.\operatorname{DOMS}\left(Y_{2}\right)\right)$.

We now state three propositions:

(74) $f_{1}+f_{2}=f_{2}+f_{1}$.

(75) $\left(f+f_{1}\right)+f_{2}=f+\left(f_{1}+f_{2}\right)$.

(76) $-\left(f_{1}+f_{2}\right)=\left(-f_{1}\right)+-f_{2}$.

Let $X_{1}, X_{2}$ be sets, let $Y_{1}, Y_{2}$ be complex-functions-membered sets, let $f$ be a partial function from $X_{1}$ to $Y_{1}$, and let $g$ be a partial function from $X_{2}$ to $Y_{2}$. The functor $f-g$ yields a function and is defined by:

(Def. 46) $\operatorname{dom}(f-g)=\operatorname{dom} f \cap \operatorname{dom} g$ and for every set $x$ such that $x \in \operatorname{dom}(f-g)$ holds $(f-g)(x)=f(x)-g(x)$.

Let $X_{1}, X_{2}$ be sets, let $Y_{1}, Y_{2}$ be complex-functions-membered sets, let $f$ be a partial function from $X_{1}$ to $Y_{1}$, and let $g$ be a partial function from $X_{2}$ to $Y_{2}$. Then $f-g$ is a partial function from $X_{1} \cap X_{2}$ to $\mathbb{C}$-PFuncs(DOMS $\left(Y_{1}\right) \cap$ $\left.\operatorname{DOMS}\left(Y_{2}\right)\right)$.

Let $X_{1}, X_{2}$ be sets, let $Y_{1}, Y_{2}$ be real-functions-membered sets, let $f$ be a partial function from $X_{1}$ to $Y_{1}$, and let $g$ be a partial function from $X_{2}$ to $Y_{2}$. Then $f-g$ is a partial function from $X_{1} \cap X_{2}$ to $\mathbb{R}$-PFuncs(DOMS $\left(Y_{1}\right) \cap$ $\left.\operatorname{DOMS}\left(Y_{2}\right)\right)$.

Let $X_{1}, X_{2}$ be sets, let $Y_{1}, Y_{2}$ be rational-functions-membered sets, let $f$ be a partial function from $X_{1}$ to $Y_{1}$, and let $g$ be a partial function from $X_{2}$ to $Y_{2}$. Then $f-g$ is a partial function from $X_{1} \cap X_{2}$ to $\mathbb{Q}-\operatorname{PFuncs}\left(\operatorname{DOMS}\left(Y_{1}\right) \cap\right.$ $\left.\operatorname{DOMS}\left(Y_{2}\right)\right)$.

Let $X_{1}, X_{2}$ be sets, let $Y_{1}, Y_{2}$ be integer-functions-membered sets, let $f$ be a partial function from $X_{1}$ to $Y_{1}$, and let $g$ be a partial function from $X_{2}$ to $Y_{2}$. Then $f-g$ is a partial function from $X_{1} \cap X_{2}$ to $\mathbb{Z}$-PFuncs(DOMS $\left(Y_{1}\right) \cap$ $\left.\operatorname{DOMS}\left(Y_{2}\right)\right)$.

One can prove the following propositions:

(77) $f_{1}-f_{2}=-\left(f_{2}-f_{1}\right)$.

(78) $-\left(f_{1}-f_{2}\right)=\left(-f_{1}\right)+f_{2}$.

(79) $\left(f+f_{1}\right)-f_{2}=f+\left(f_{1}-f_{2}\right)$.

(80) $\left(f-f_{1}\right)+f_{2}=f-\left(f_{1}-f_{2}\right)$.

(81) $f-f_{1}-f_{2}=f-\left(f_{1}+f_{2}\right)$.

(82) $f-f_{1}-f_{2}=f-f_{2}-f_{1}$.

Let $X_{1}, X_{2}$ be sets, let $Y_{1}, Y_{2}$ be complex-functions-membered sets, let $f$ be a partial function from $X_{1}$ to $Y_{1}$, and let $g$ be a partial function from $X_{2}$ to $Y_{2}$. 
The functor $f \cdot g$ yields a function and is defined by:

(Def. 47) $\operatorname{dom}(f \cdot g)=\operatorname{dom} f \cap \operatorname{dom} g$ and for every set $x$ such that $x \in \operatorname{dom}(f \cdot g)$ holds $(f \cdot g)(x)=f(x) g(x)$.

Let $X_{1}, X_{2}$ be sets, let $Y_{1}, Y_{2}$ be complex-functions-membered sets, let $f$ be a partial function from $X_{1}$ to $Y_{1}$, and let $g$ be a partial function from $X_{2}$ to $Y_{2}$. Then $f \cdot g$ is a partial function from $X_{1} \cap X_{2}$ to $\mathbb{C}-\operatorname{PFuncs}\left(\operatorname{DOMS}\left(Y_{1}\right) \cap\right.$ $\left.\operatorname{DOMS}\left(Y_{2}\right)\right)$.

Let $X_{1}, X_{2}$ be sets, let $Y_{1}, Y_{2}$ be real-functions-membered sets, let $f$ be a partial function from $X_{1}$ to $Y_{1}$, and let $g$ be a partial function from $X_{2}$ to $Y_{2}$. Then $f \cdot g$ is a partial function from $X_{1} \cap X_{2}$ to $\mathbb{R}-\operatorname{PFuncs}\left(\operatorname{DOMS}\left(Y_{1}\right) \cap\right.$ $\left.\operatorname{DOMS}\left(Y_{2}\right)\right)$.

Let $X_{1}, X_{2}$ be sets, let $Y_{1}, Y_{2}$ be rational-functions-membered sets, let $f$ be a partial function from $X_{1}$ to $Y_{1}$, and let $g$ be a partial function from $X_{2}$ to $Y_{2}$. Then $f \cdot g$ is a partial function from $X_{1} \cap X_{2}$ to $\mathbb{Q}$-PFuncs $\left(\operatorname{DOMS}\left(Y_{1}\right) \cap\right.$ $\left.\operatorname{DOMS}\left(Y_{2}\right)\right)$.

Let $X_{1}, X_{2}$ be sets, let $Y_{1}, Y_{2}$ be integer-functions-membered sets, let $f$ be a partial function from $X_{1}$ to $Y_{1}$, and let $g$ be a partial function from $X_{2}$ to $Y_{2}$. Then $f \cdot g$ is a partial function from $X_{1} \cap X_{2}$ to $\mathbb{Z}$-PFuncs(DOMS $\left(Y_{1}\right) \cap$ $\left.\operatorname{DOMS}\left(Y_{2}\right)\right)$.

Let $X_{1}, X_{2}$ be sets, let $Y_{1}, Y_{2}$ be natural-functions-membered sets, let $f$ be a partial function from $X_{1}$ to $Y_{1}$, and let $g$ be a partial function from $X_{2}$ to $Y_{2}$. Then $f \cdot g$ is a partial function from $X_{1} \cap X_{2}$ to $\mathbb{N}$-PFuncs(DOMS $\left(Y_{1}\right) \cap$ $\left.\operatorname{DOMS}\left(Y_{2}\right)\right)$.

We now state several propositions:

(83) $f_{1} \cdot f_{2}=f_{2} \cdot f_{1}$.

(84) $\left(f \cdot f_{1}\right) \cdot f_{2}=f \cdot\left(f_{1} \cdot f_{2}\right)$.

(85) $\left(-f_{1}\right) \cdot f_{2}=-f_{1} \cdot f_{2}$.

(86) $f_{1} \cdot-f_{2}=-f_{1} \cdot f_{2}$.

(87) $f \cdot\left(f_{1}+f_{2}\right)=f \cdot f_{1}+f \cdot f_{2}$.

(88) $\left(f_{1}+f_{2}\right) \cdot f=f_{1} \cdot f+f_{2} \cdot f$.

(89) $f \cdot\left(f_{1}-f_{2}\right)=f \cdot f_{1}-f \cdot f_{2}$.

(90) $\left(f_{1}-f_{2}\right) \cdot f=f_{1} \cdot f-f_{2} \cdot f$.

Let $X_{1}, X_{2}$ be sets, let $Y_{1}, Y_{2}$ be complex-functions-membered sets, let $f$ be a partial function from $X_{1}$ to $Y_{1}$, and let $g$ be a partial function from $X_{2}$ to $Y_{2}$. The functor $f / g$ yields a function and is defined by:

(Def. 48) $\operatorname{dom}(f / g)=\operatorname{dom} f \cap \operatorname{dom} g$ and for every set $x$ such that $x \in \operatorname{dom}(f / g)$ holds $(f / g)(x)=f(x) / g(x)$.

Let $X_{1}, X_{2}$ be sets, let $Y_{1}, Y_{2}$ be complex-functions-membered sets, let $f$ be a partial function from $X_{1}$ to $Y_{1}$, and let $g$ be a partial function from $X_{2}$ 
to $Y_{2}$. Then $f / g$ is a partial function from $X_{1} \cap X_{2}$ to $\mathbb{C}-\operatorname{PFuncs}\left(\operatorname{DOMS}\left(Y_{1}\right) \cap\right.$ $\left.\operatorname{DOMS}\left(Y_{2}\right)\right)$.

Let $X_{1}, X_{2}$ be sets, let $Y_{1}, Y_{2}$ be real-functions-membered sets, let $f$ be a partial function from $X_{1}$ to $Y_{1}$, and let $g$ be a partial function from $X_{2}$ to $Y_{2}$. Then $f / g$ is a partial function from $X_{1} \cap X_{2}$ to $\mathbb{R}$-PFuncs(DOMS $\left(Y_{1}\right) \cap$ $\left.\operatorname{DOMS}\left(Y_{2}\right)\right)$.

Let $X_{1}, X_{2}$ be sets, let $Y_{1}, Y_{2}$ be rational-functions-membered sets, let $f$ be a partial function from $X_{1}$ to $Y_{1}$, and let $g$ be a partial function from $X_{2}$ to $Y_{2}$. Then $f / g$ is a partial function from $X_{1} \cap X_{2}$ to $\mathbb{Q}$-PFuncs $\left(\operatorname{DOMS}\left(Y_{1}\right) \cap\right.$ $\left.\operatorname{DOMS}\left(Y_{2}\right)\right)$.

One can prove the following propositions:

(91) $\left(-f_{1}\right) / f_{2}=-f_{1} / f_{2}$.

(92) $f_{1} /-f_{2}=-f_{1} / f_{2}$.

(93) $\quad\left(f \cdot f_{1}\right) / f_{2}=f \cdot\left(f_{1} / f_{2}\right)$.

(94) $\left(f / f_{1}\right) \cdot f_{2}=\left(f \cdot f_{2}\right) / f_{1}$.

(95) $f / f_{1} / f_{2}=f /\left(f_{1} \cdot f_{2}\right)$.

(96) $\left(f_{1}+f_{2}\right) / f=f_{1} / f+f_{2} / f$.

(97) $\left(f_{1}-f_{2}\right) / f=f_{1} / f-f_{2} / f$.

\section{REFERENCES}

[1] Grzegorz Bancerek. The ordinal numbers. Formalized Mathematics, 1(1):91-96, 1990.

[2] Czesław Byliński. Functions and their basic properties. Formalized Mathematics, 1(1):5565, 1990.

[3] Czesław Byliński. Functions from a set to a set. Formalized Mathematics, 1(1):153-164, 1990.

[4] Czesław Byliński. Some basic properties of sets. Formalized Mathematics, 1(1):47-53, 1990.

[5] Andrzej Kondracki. Basic properties of rational numbers. Formalized Mathematics, 1(5):841-845, 1990

[6] Andrzej Trybulec. Tuples, projections and Cartesian products. Formalized Mathematics, 1(1):97-105, 1990.

[7] Andrzej Trybulec. On the sets inhabited by numbers. Formalized Mathematics, 11(4):341$347,2003$.

[8] Michał J. Trybulec. Integers. Formalized Mathematics, 1(3):501-505, 1990.

[9] Zinaida Trybulec. Properties of subsets. Formalized Mathematics, 1(1):67-71, 1990.

[10] Edmund Woronowicz. Relations and their basic properties. Formalized Mathematics, 1(1):73-83, 1990

[11] Edmund Woronowicz. Relations defined on sets. Formalized Mathematics, 1(1):181-186, 1990. 